Cahiers $d u$ MONDE RUSSE

\section{Cahiers du monde russe}

Russie - Empire russe - Union soviétique et États indépendants

$48 / 4 \mid 2007$

Varia

\title{
A. N. Čistikov, Partijno-gosudarstvennaja bjurokratija severo-zapada sovetskoj rossii 1920-h godov
}

Alexandre Sumpf

\section{(2) OpenEdition}

Journals

Édition électronique

URL : https://journals.openedition.org/monderusse/6076

DOI : 10.4000/monderusse.6076

ISSN : $1777-5388$

Éditeur

Éditions de l'EHESS

Édition imprimée

Date de publication : 2 décembre 2007

Pagination : 702-705

ISBN : 978-2-7132-2148-4

ISSN : $1252-6576$

Référence électronique

Alexandre Sumpf, «A. N. Čistikov, Partijno-gosudarstvennaja bjurokratija severo-zapada sovetskoj rossii 1920-h godov », Cahiers du monde russe [En ligne], 48/4 | 2007, mis en ligne le 03 juillet 2009, consulté le 04 septembre 2022. URL : http://journals.openedition.org/monderusse/6076 ; DOI : https://doi.org/10.4000/monderusse.6076

Ce document a été généré automatiquement le 4 septembre 2022.

Tous droits réservés 


\title{
A. N. Čistikov, Partijno- gosudarstvennaja bjurokratija severo-zapada sovetskoj rossii 1920- $\mathrm{h}$ godov
}

\author{
Alexandre Sumpf
}

\section{RÉFÉRENCE}

\section{A. N. ČISTIKOV, Partijno-gosudarstvennaja bjurokratija Severo-Zapada Sovetskoj}

Rossii 1920-h godov [La bureaucratie de l'État et du parti dans la région du Nord-Ouest de la Russie soviétique dans les années 1920]. Saint-Pétersbourg : Evropejskij dom, 2007, $292 \mathrm{p}$.

1 Ces dernières années, en Russie, les travaux de T.P. Koržihina et E.G. Gimpelson ont ouvert la voie à des recherches centrées sur l'époque de la NEP, considérée depuis les écrits de Moshe Lewin comme la période de «formation du système soviétique». A.N. Čistikov, professeur à l'Université européenne de Saint-Pétersbourg, s'inscrit dans cette lignée avec son ouvrage consacré à la « bureaucratie » de la région du Nord-Ouest, future oblast每 de Leningrad. L'étude limitée à cette région livre des enseignements généralisables à l'Union soviétique.

Le livre comprend six parties assez équilibrées. On regrette toutefois le nombre restreint de sous-parties où devrait apparaitre le détail des questions abordées. Cela dit, la démarche est convaincante. L'auteur traite tout d'abord de la structure de l'appareil de l'État et du parti dans les régions pendant la NEP (I), puis des formes et des méthodes d'action de l'appareil du parti (II), de la formation des cadres de la nouvelle bureaucratie (III), du décompte et de la répartition des cadres dirigeants (IV), des responsables du Nord-Ouest dans les années 1920 (V), et enfin du mode de vie de la bureaucratie de l'État et du parti (VI). 
3 Le premier chapitre livre la trame générale de l'évolution de l'administration du pays, qui passe d'abord par la «liquidation» des institutions de l'époque tsariste et l'établissement d'un pouvoir centralisé. Au tournant de la NEP, les instances choisies (comités exécutifs) prennent le pas sur les instances élues et closent ainsi l'épisode révolutionnaire. Dans le second chapitre, Čistikov montre que cet effort de centralisation est relatif: l'autonomie des institutions locales résiste jusqu'au Grand Tournant. Les tournées épisodiques des instructeurs ou les rapports irréguliers n'assurent qu'un lien ténu entre centre et périphéries régionales - d'autant plus que ledit «centre » oscille entre deux politiques opposées. Faut-il recueillir la totalité des informations locales (enquête) ou exiger un résumé immédiatement utilisable (contrôle); faire respecter l'échelle hiérarchique établie à grand-peine ou instaurer un rapport plus direct entre cellules locales et direction centrale ? Cela est-il possible quand les mutations fréquentes imposées aux cadres locaux gênent la consolidation des positions du parti?

Ces relations centre/périphérie (III) passent surtout par des individus qui forment, selon les termes de l'auteur, un « conglomérat » de fonctionnaires d'Ancien Régime, de cadres nouvellement formés et de révolutionnaires professionnels. Čistikov néglige le remarquable cumul des fonctions par un même individu, qui solidarise ces relations à la fois professionnelles et interpersonnelles, localement comme au centre. Or les problèmes de recrutement sont récurrents; plus on avance dans la décennie, plus les dirigeants bolcheviks déplorent la baisse du niveau d'instruction et de la qualité de l'engagement politique. Mais cela n'a pas obéré la mise en place d'une bureaucratie où fidélité prime sur efficacité.

5 C'est sans doute pour cela que Čistikov s'attarde si peu sur les raisons de ce recrutement difficile- ou sur les motivations de ceux qui s'engagent dans l'administration soviétique - et se focalise sur la machinerie. L'un des passages les plus innovants de l'ouvrage est l'étude minutieuse des vœux d'affectation et des destinations finales des étudiants de l'université communiste Zinov'ev, équivalent leningradois de l'université Sverdlov étudiée par Michael David-Fox. Ce dernier avait privilégié la composition sociale - une majorité de jeunes "employés » ayant adhéré au parti pendant la guerre civile ou juste avant d'entrer à l'université -, l'éthique et le byt, sans analyser la mise à l'épreuve du groupe soudé par une culture collective après sa dissolution par l'envoi sur place des nouveaux « cadres ». Pour sa part, Čistikov dégage une logique d'attribution liée aux besoins, où, horizontalement (entre instances territoriales de même rang), prime une volonté d'équilibre géographique imposée « d'en haut » plutôt qu'obtenue « d'en bas».

6 L'année 1924 apparaît ici comme un tournant, comme c'est souvent le cas lorsqu'on étudie l'administration soviétique en formation. Dans une région déjà bien pourvue, les derniers fonctionnaires formés sont alors contraints de s'employer au fin fond des campagnes. Ils y occupent des postes de base, souvent dans l'agit-prop - ou l'éducation politique, que Čistikov ne distingue pas, pas plus d'ailleurs qu'il n'analyse les statuts officiels des différentes institutions. En revanche, il utilise à bon escient des sources rarement exploitées : formulaires personnels (ankety), correspondances, évaluations (harakteristiki) et auto-évaluations (samoocenki) montrent que les aspects psychologiques sont aussi importants que les catégories sociales, alors que conviction et " conscience " politiques sont reléguées à l'arrière-plan. Cela dit, ces documents ouvrent un véritable accès aux opinions des individus : en les relisant dans cette optique, on dégagerait sûrement plus qu'un simple effort de mise en conformité aux exigences supposées de la hiérarchie. 
7 Pour asseoir cette thèse, Čistikov aurait pu se contenter de l'étude minutieuse des commissions d'attestations des gubkomy du parti, qui avalisent les nominations, établissent les nomenclatures et règlent les conflits entre groupes adverses - fréquents dans cette région capitale. L'auteur montre qu'une promotion sociale souvent limitée à l'échelle territoriale et un faible niveau initial de recrutement ne motivent pas l'attrait de ces fonctions. Cette partie (IV) de l'ouvrage souffre de ne pas s'intéresser vraiment aux dynamiques au niveau local qui expliqueraient le fonctionnement général d'une machine trop souvent décrite à l'arrêt.

8 Un corpus de 1012 hauts fonctionnaires régionaux de la première moitié des années 1920 (V) est présenté en 74 tableaux et 14 sections. L'analyse détaillée permet de dresser un portrait type des employés de l'administration: $1 / 3$ est sans parti et $1 / 3$ a adhéré pendant la guerre civile ; les $2 / 3$ appartiennent à la catégorie sociale des « employés »; la moitié a entre 30 et 39 ans; $1 / 3$ seulement possède un niveau d'instruction secondaire. Peu de paysans et d'ouvriers, donc, même si un certain nombre de communistes de la nouvelle vague parviennent à des postes de responsabilité de plus en plus nombreux.

Le dernier chapitre, consacré au mode de vie, est envisagé surtout du point de vue de la rémunération et se révèle le plus faible de l'ouvrage. Les salaires sont évoqués en chiffres précis, mais parcellaires. L'auteur ne produit pas une série statistique, mais doit se contenter de chiffres datant de 1921, cette année de mise en place du système administratif qui est souvent bien représentée dans les archives. En outre, les salaires ne sont pas mis en relation avec le coût de la vie, alors que les travaux de Elena Osokina y invitent. D'ailleurs, les notes n'indiquent aucun ouvrage occidental : l'auteur a-t-il choisi de se passer des apports de l'historiographie ? Cela expliquerait pourquoi la hiérarchie du razrjad soviétique, longuement détaillée, n'est pas comparée aux činy de l'administration tsariste, et que son fonctionnement n'est pas expliqué.

Voilà pourquoi la thèse du rôle central des prestations en nature, probablement juste, ne paraît pas suffisamment établie. Selon Čistikov, tout ce système s'est mis en place durant la guerre civile, puis s'est consolidé et élargi à la faveur de la NEP, quand il a fallu fidéliser les nouveaux cadres du régime. À la fois symboles d'appartenance et produits d'appel, ces avantages concrets creusent le fossé entre les fonctionnaires et le reste de la population, tout en figeant les hiérarchies à l'intérieur de l'administration. Les déviations comportementales, l'alcoolisme au premier chef, sont étudiées du seul point de vue répressif et non dans une perspective anthropologique ou sociologique.

11 En d'autres termes, cette étude réellement novatrice est riche en informations détaillées, fructueuse par l'invention des sources et soulève de nombreuses questions; mais l'auteur, assez timide dans ses conclusions, ne se démarque guère de l'histoire rénovée des institutions initiée par T.P. Koržihina. 